\title{
Patients with spinal cord injury: mental status, health, behaviour and rehabilitation outcome
}

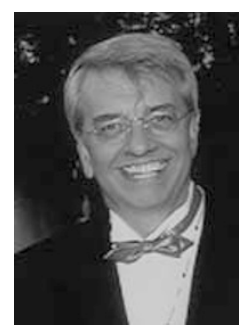

\author{
JJ Wyndaele, Editor \\ Antwerp University Hospital, Antwerp, Belgium \\ E-mail: spinalcord@uza.be
}

Dear Spinal Cord reader,

In this April issue, Wadsworth et al. review the evidence for using an abdominal binder on breathing, speech and cardiovascular function in people who have suffered a spinal cord injury (SCI). They found some evidence that using an abdominal binder improves vital capacity, but decreases functional residual capacity when assuming the sitting or tilted position. They found the overall quality of related studies to be poor and available evidence not yet convincing. Further studies that use more methodologically rigorous designs are required.

Several contributions look into specific patient groups. Kennedy et al. investigated rehabilitation outcomes of spinal-cord-injured patients with pre-existing mental health disorders. They found, with proper management, outcomes do not differ significantly from those in patients who do not have mental health disorders.

In a prospective cross-sectional study, Gupta et al. studied epidemiology, complications, and neurological and functional outcomes in non-traumatic spinal cord lesions after in-patient rehabilitation. They found female patients, paraplegia and incomplete cord lesions to be more common in this type of lesions. They described significant neurological and functional recovery with rehabilitation intervention.

Lannem et al. investigated the role of physical exercise, perceived exercise mastery and fitness on life satisfaction of a sample of individuals with incomplete SCI in Norway. Those who exercised regularly experienced a significantly higher life satisfaction and perceived exercise fitness, but lower perceived exercise mastery than their inactive peers. Perceived exercise fitness was the psychological variable that contributed meaningfully to life satisfaction in this study.

In an interesting study from British Columbia, Tawashi et al. found activities of daily living to be a large component for physical activity among individuals with SCI. It appeared that greater physical activity is associated with fewer secondary complications (pain, fatigue and depression).

Walters et al. estimated the prevalence of inadequate dietary intakes in community-dwelling men and women with chronic SCI. They found numerous nutrient inadequacies, which have important implications for clinical dietetic practice in the SCI population.

Bowel management is evaluated by Coggrave et al. in community-dwelling SCI individuals by postal questionnaires. Their findings show that managing SCI bowel function in the community is complex, time consuming and remains conservative. Further exploration of bowel management is needed to reduce impact, minimise side effects and increase the choice of available management strategies.

Other manuscripts are equally interesting: unmyelinated fibres in human spinal ventral roots (Ko et al.), diagnostic work-up of patients with transverse myelitis (Sellner et al.), benefits and risks associated with surgically improving arm function (Anderson et al.) and experience with SCI rehabilitation in Dutch and Flemish rehabilitation centres (Osterthun et al.).

Two interesting case reports and correspondence regarding earlier publications are also included.

Spinal Cord (2009) 47, 273; doi:10.1038/sc.2009.25 